\title{
Резонансное поглощение электромагнитного излучения в монослое фосфорена
}

\author{
(C) В.В. Карпунин ${ }^{1}$, В.А. Маргулис ${ }^{2}$ \\ ${ }^{1}$ Мордовский государственный педагогический институт им. М.Е. Евсевьева, \\ 430007 Саранск, Россия \\ ${ }^{2}$ Мордовский государственный университет им. Н.П. Огарева, \\ 430005 Саранск, Россия \\ E-mail: karpuninvv@mail.ru \\ (Поступила в Редакцию 2 июля 2018 г. \\ В окончательной редакции 22 октября 2018 г. \\ Принята к публикации 22 октября 2018 г.)
}

\begin{abstract}
Найден коэффициент поглощения электромагнитного излучения в монослое фосфорена, помещенном в магнитное поле. Рассмотрен вырожденный и невырожденный электронный газ. Установлены резонансные зависимости коэффициента поглощения от частоты излучения и от приложенного магнитного поля. Учет рассеяния электронов на ионизованной примеси приводит к осцилляционным зависимостям коэффициента поглощения от частоты излучения и внешнего магнитного поля. Показан резонансный характер кривой поглощения. Найдены условия резонансов и положения резонансных пиков.
\end{abstract}

DOI: 10.21883/FTP.2019.04.47443.8944

\section{1. Введение}

Фосфорен, одиночный слой черного фосфора, в последнее время привлекает значительное внимание благодаря своим необычным электронным свойствам. В [1] теоретически исследуются уровни Ландау и магнитотранспортные свойства фосфорена, на который наложено перпендикулярное магнитное поле. Спектр электронов получен в рамках эффективного $\mathbf{k} \cdot \mathbf{p}$ гамильтониана и модели сильной связи. В слабых магнитных полях показано, что уровни Ландау линейно зависят как от индекса $n$, так и от магнитного поля $B$, т.е. аналогичны уровням обычного полупроводникового двумерного электронного газа. Показано, что расщепления уровней Ландау для зоны проводимости и валентной зоны различны, а волновые функции, сильно анизотропны из-за различных анизотропных эффективных масс.

В [2] получена и обсуждена зонная структура монослоя фосфорена в присутствии перпендикулярного к плоскости слоя магнитного поля. Проведены оценки магнитооптической, холловской и продольной оптических проводимостей в зависимости от температуры, магнитного поля и энергии Ферми, и показано, что они сильно зависят от величины магнитного поля.

В [3] вводится низкоэнергетический гамильтониан однослойного и двухслойного черного фосфора, который описывает электронные состояния в окрестности Г точки зоны Бриллюэна. Найдены выражения для уровней Ландау в зависимости от магнитного поля. В случае двухслойного черного фосфора исследуется также эффект внешнего смещения в электронной запрещенной зоне. Результаты демонстрируют сильно анизотропный характер черного фосфора (ЧФ) и, в частности, для двухслойного ЧФ, наличие смещения позволяет получить переход полупроводник-металл.
Используя приближение сильной связи с учетом спин-орбитального взаимодействия, в [4] предсказывается, в присутствии осевых деформаций, топологический фазовый переход в электронной зонной структуре фосфорена.

Теория оптического солитона самоиндуцированной прозрачности в монослое черного фосфора (фосфорена) построена в [5]. Получены явные аналитические выражения для поверхностного солитона в фосфорене и других анизотропных двумерных материалах.

В [6] систематически изучены структурные, электронные и магнитные свойства голубого фосфорена, легированного атомами групп IV и VI, включая C, Si, Ge, $\mathrm{Sn}, \mathrm{O}, \mathrm{S}, \mathrm{Se}$ и Те. Обнаружено, что легированные $\mathrm{C}, \mathrm{Si}$, $\mathrm{Ge}$ и $\mathrm{O}$ системы являются непрямыми запрещенными полупроводниками, в то время как легированные $\mathrm{Sn}, \mathrm{S}$, $\mathrm{Se}$ и Tе системы имеют металлические свойства.

Структурные, электронные и магнитные свойства фосфорена, легированного атомами переходных металлов были исследованы в рамках метода функционала плотности, основанного на расчетах спиновой поляризации [7]. Структурные, электронные и магнитные свойства фосфорена, легированного $\mathrm{Eu}$, с различными концентрациями были исследованы в [8].

Из первых принципов проведены вычисления, основанные на методе функционала плотности в [9]. Были проведены исследования магнитных свойств фосфорена. Установлено, что вакансии или внешняя деформация не приводят к магнетизму в фосфорене, однако взаимодействие между вакансиями и внешним напряжением может привести к магнетизму.

Используя расчеты из первых принципов, рассмотрено влияние графена и нитрида бора (НБ) на электронные свойства фосфорена [10]. Фосфорен под влиянием графена оказывается металлическим, в то время как под 
влиянием НБ фосфорен представляет собой полупроводник с умеренной шириной запрещенной зоны 1.02 эВ.

В [11] исследована электронная структура и магнетизм зигзагообразных синих фосфореновых нанолент с использованием техники вычислений на основе функционала плотности. Исследовались изменения ширины от 1.5 до 5 нм. Расчеты из первых принципов основных спин-орбитальных и спиновых релаксационных свойств фосфорена проведены в [12].

Анизотропное оптическое поглощение в монослое фосфорена с учетом спин-орбитального взаимодействия Рашбы теоретически исследовано в [13]. Проведен численный расчет оптического поглощения электронами.

В [14] теоретически изучаются магнитооптические свойства монослоя фосфорена, находящегося в перпендикулярном плоскости магнитном поле. Проведены оценки линейного, нелинейного 3-го порядка и полного коэффициента поглощения, а также относительного показателя преломления как функции энергии фотона и магнитного поля. Оценки показывают, что эти величины сильно зависят от магнитного поля.

В [15] исследуется электронная структура монослоя черного фосфора с использованием метода функционала плотности как в случае с приложенным электрическим полем, так и без него.

В [16] изучены электронные и оптические свойства однослойных квантовых точек фосфорена, имеющих различную форму. По сравнению с графеновыми квантовыми точками, в фосфореновых кластерах, имеющих одинаковую форму и размер, имеется набор граничных состояний с энергиями, расположенными вокруг уровня Ферми.

В [17] рассмотрено возникновение атомных вакансий в краевой структуре зигзагообразных фосфореновых и графеновых наноуглеродов. Для различных концентраций этих краевых вакансий исследовалось их влияние на металлические свойства. Расчеты выполнялись для разных размеров элементарной ячейки. Кроме того, при меньших размерах рассмотрено и влияние однородного магнитного поля.

В [18] сообщается об электронной структуре и спектрах оптического поглощения монослоя черного фосфора, полученных из первых принципов.

В [19] исследовалась электронная и магнитная структура зигзагообразных фосфореновых нанолент различной ширины.

Изучению электронных свойств двумерного электронного газа в многослойных слоях черного фосфора в перпендикулярном магнитном поле посвящена работа [20]. Показано, что резонансные структуры в проводимости переменного тока имеют красное смещение с увеличением легирования из-за межзонной связи $\gamma$. Это возникает из-за дополнительного поправочного члена в энергетическом спектре Ландау, пропорционального $n^{2} \gamma^{2}(n-$ индекс Ландау), до 2-го порядка в $\gamma$.

Цель настоящей работы заключается в нахождении и анализе коэффициента поглощения (КП) электро- магнитного излучения электронным газом в монослое фосфорена. Рассмотрен случай внутризонных переходов между уровнями как невырожденного, так и вырожденного электронного газа. В первом разделе расмотрены прямые электронные переходы под влиянием внешнего электромагнитного излучения. Во втором разделе расчет проведен с учетом рассеяния электронов на ионизованной примеси.

\section{2. Коэффициент поглощения электромагнитного излучения}

Рассмотрим квазиодномерный электронный газ в монослое фосфорена. Гамильтониан такой системы определяется выражением [1,2]

$$
H=\left(\begin{array}{cc}
E_{c}+\left(\alpha^{\prime} \Pi_{x}^{2}+\beta \Pi_{y}^{2}\right) / 2 & 0 \\
0 & E_{v}-\left(\lambda^{\prime} \Pi_{x}^{2}-\eta \Pi_{y}^{2}\right) / 2
\end{array}\right),
$$

где $E_{c}$ соответствует границе зоны проводимости, $E_{v}$ соответствует границе валентной зоны, $\alpha^{\prime}=\alpha+\gamma^{2} / E_{g}$, $\lambda^{\prime}=\lambda+\gamma^{2} / E_{g}, \quad \gamma=8.5 \cdot 10^{5} \mathrm{~m} / \mathrm{c}, \quad \alpha=1 / m_{c x}, \quad \beta=1 / m_{c y}$, $\lambda=1 / m_{v x}, \quad \eta=1 / m_{v y}, \quad E_{g}=E_{c}-E_{v}=1.52$ эВ,$\quad \boldsymbol{\Pi}=$ $=\mathbf{p}+\frac{e}{c} \mathbf{A}-$ оператор обобщенного импульса.

Энергетический спектр собственных состояний электронов в магнитном поле может быть записан следующим образом $[1,2]$ :

$$
E_{n, s}=E_{s}+s\left(n+\frac{1}{2}\right) \hbar \omega_{s}, \quad n=0,1,2,3 \ldots,
$$

где $s \pm 1$ соответствует зоне проводимости и валентной зоне, $E_{s}=E_{+}=E_{c}$ - для зоны проводимости, $\omega_{+}=\omega_{c}^{\prime}=e B /\left(m_{c x}^{\prime} m_{c y}\right)^{1 / 2} c=2.657 \omega_{e}-$ модифицированная циклотронная частота, $\omega_{e}=e B / m_{e} c$.

Если использовать калибровку Ландау $\mathbf{A}=\left(-B_{y}, 0,0\right)$, то соответствующая гамильтониану (1) и энергетическому спектру (2) волновая функция для электронов зоны проводимости имеет вид $[1,2]$

$$
\psi_{n,+}(x, y)=\frac{\exp \left(i k_{x} x\right)}{\sqrt{L_{x}}}\left(\begin{array}{c}
\phi_{n}\left(y_{c}\right) \\
0
\end{array}\right)
$$

где $y_{c}=\left(y-y_{0}\right) / l_{c}, y_{0}=l_{B}^{2} k_{x}, l_{B}=\sqrt{\hbar c / e B}$ - магнитная длина, $l_{c}=\sqrt{\hbar / m_{c y} \omega_{c}^{\prime}}, \phi_{n}\left(y_{c}\right)$ - осцилляторные функции.

Найдем матричный элемент оператора электронфотонного взаимодействия. Оператор электрон-фотонного взаимодействия при направлении вектора поляризации фотона по оси $у$ имеет вид

$$
H_{R}=\frac{i e \hbar}{m_{c y}} \sqrt{\frac{2 \pi \hbar N_{f}}{\varepsilon \omega}} \frac{\partial}{\partial y},
$$

где $N_{f}$ - число фотонов, $\varepsilon-$ диэлектрическая проницаемость. 
Матричный элемент $H_{R}$, как следует из (3) и (4), может быть записан в виде

$$
\begin{array}{r}
\left\langle n, k_{x}, 0\left|H_{R}\right| n^{\prime}, k_{x}^{\prime},-\mathbf{f}\right\rangle=\frac{-i e \hbar}{m_{c y} l_{c}} \sqrt{\frac{2 \pi \hbar N_{f}}{\varepsilon \omega}} \delta_{k_{x}, k_{x}^{\prime}} \\
\times\left(\sqrt{\frac{n+1}{2}} \delta_{n, n^{\prime}-1}-\sqrt{\frac{n}{2}} \delta_{n, n^{\prime}+1}\right),
\end{array}
$$

$\mathbf{f}$ - волновой вектор фотона.

Введем коэффициент поглощения $\Gamma(\omega)$, используя теорию возмущений. Тогда аналогично случаю $2 D$ электронного газа [21] можно записать для невырожденного электронного газа в нашем случае

$$
\Gamma=\frac{2 \pi \sqrt{\varepsilon}}{c \hbar N_{f}} \sum_{\alpha} \sum_{\alpha^{\prime}} f_{0}\left(E_{\alpha}\right)\left|\left\langle\alpha\left|H_{R}\right| \alpha^{\prime}\right\rangle\right|^{2} \delta\left(E_{\alpha}-E_{\alpha^{\prime}}+\hbar \omega\right),
$$

где $\alpha, \alpha^{\prime}-$ квантовые числа начального и конечного состояний.

Рассмотрим КП в случае невырожденного электронного газа. Нахождение КП проведем с использованием подхода, примененного в [21]. Тогда коэффициент поглощения запишется в виде

$$
\begin{aligned}
\Gamma= & \frac{2 \pi \sqrt{\varepsilon}}{c \hbar N_{f}} \sum_{k_{x}} \sum_{k_{x}^{\prime}} \sum_{n} \sum_{n^{\prime}} f_{0}\left(E_{n,+1}\right) \mid\left\langle n, k_{x}, 0\right| \\
& \times\left. H_{R}\left|n^{\prime}, k_{x}^{\prime}-\mathbf{f}\right\rangle\right|^{2} \delta\left(E_{n}-E_{n^{\prime}}+\hbar \omega\right),
\end{aligned}
$$

где $f_{0}\left(E_{n,+1}\right)$ - функция распределения невырожденного электронного газа, нормировочный множитель в $f_{0}\left(E_{n}\right)$ определяется по формуле

$$
\sum_{n} A \exp \left(-E_{c} / T\right) \exp \left(-\hbar \omega_{c}^{\prime}(n+1 / 2) / T\right)=n_{e},
$$

где $n_{e}-$ концентрация электронов в монослое.

Нормировочный множитель имеет вид

$$
A=2 n_{e} \sinh \left(\frac{\hbar \omega_{c}^{\prime}}{2 T}\right) \exp \left(E_{c} / T\right) .
$$

В итоге (7) приводит к следующему выражению для КП:

$$
\begin{aligned}
\Gamma & =\frac{2 \pi \sqrt{\varepsilon}}{c \hbar N_{f}}\left(\frac{e \hbar}{m_{c y} l_{c}}\right)^{2} \frac{2 \pi \hbar N_{f}}{\epsilon \omega} 2 n_{e} \sinh \left(\frac{\hbar \omega_{c}^{\prime}}{2 T}\right) \\
& \times \sum_{k_{x}} \sum_{k_{x}^{\prime}} \delta_{k_{x}, k_{x}^{\prime}} \sum_{n} \sum_{n^{\prime}} \exp \left(-\hbar \omega_{c}^{\prime}(n+1 / 2) / T\right) \\
& \times\left(\frac{n+1}{2} \delta_{n, n^{\prime}-1}-\frac{n}{2} \delta_{n, n^{\prime}+1}\right) \delta\left(E_{n,+1}-E_{n^{\prime},+1}+\hbar \omega\right) .
\end{aligned}
$$

Здесь $\delta(x)-\delta$-функция Дирака. Чтобы учесть размытие резонанса рассеянием, введем лоренцево уширение по формуле, заменяя $\delta\left(E_{n,+1}-E_{n^{\prime},+1}+\hbar \omega\right)$ на $\left(\pi \tau_{0}\right)^{-1} /\left(\tau_{0}^{-2}+\left(\left(E_{m^{\prime}+1}-E_{m^{\prime}}\right) \hbar^{-1}+\omega\right)^{2}\right), \tau_{0}$ - феноменологическое время релаксации.
Обозначим

$$
\Gamma_{0}=\frac{4 \pi e^{2} n_{e} \tau_{0}}{c \sqrt{\epsilon} m_{c y}}
$$

тогда

$$
\begin{aligned}
\frac{\Gamma}{\Gamma_{0}}= & \sinh \left(\frac{\hbar \omega_{c}^{\prime}}{2 T}\right) \\
& \times \frac{\omega_{c}^{\prime}}{\omega}\left(\frac{\exp \left(-\hbar \omega_{c}^{\prime} / 2 T\right)}{\left(1-\exp \left(-\hbar \omega_{c}^{\prime} / T\right)\right)^{2}} \frac{1}{1+\tau_{0}^{2}\left(\left(\omega-\omega_{c}^{\prime}\right)^{2}\right)}\right. \\
& \left.-\frac{\exp \left(-3 \hbar \omega_{c}^{\prime} / 2 T\right)}{\left(1-\exp \left(-\hbar \omega_{c}^{\prime} / T\right)\right)^{2}} \frac{1}{1+\tau_{0}^{2}\left(\left(\omega+\omega_{c}^{\prime}\right)^{2}\right)}\right) .
\end{aligned}
$$

Вычисление $\Gamma(\omega)$ в случае вырожденного электронного газа проведем по аналогии с [22]:

$$
\begin{gathered}
\Gamma=\frac{2 \pi \sqrt{\varepsilon}}{c \hbar N_{f}} \sum_{\alpha} \sum_{\alpha^{\prime}} f_{0}\left(E_{\alpha}\right)\left(1-f_{0}\left(E_{\alpha^{\prime}}\right)\right) \\
\times\left|\left\langle\alpha\left|H_{R}\right| \alpha^{\prime}\right\rangle\right|^{2} \delta\left(E_{\alpha}-E_{\alpha^{\prime}}+\hbar \omega\right) .
\end{gathered}
$$

В случае вырожденного электронного газа для $\Gamma(\omega)$ получим в итоге выражение

$$
\begin{aligned}
& \frac{\Gamma}{\Gamma_{0}}=\frac{\omega_{c}^{\prime}}{\omega} \\
& \times \sum_{n}\left(f\left(E_{n,+1}\right)\left(1-f\left(E_{n+1,+1}\right)\right) \frac{n+1}{2} \frac{1}{1+\tau_{0}^{2}\left(\left(\omega-\omega_{c}^{\prime}\right)^{2}\right)}\right. \\
& \left.-f\left(E_{n,+1}\right)\left(1-f\left(E_{n-1,+1}\right)\right) \frac{n}{2} \frac{1}{1+\tau_{0}^{2}\left(\left(\omega+\omega_{c}^{\prime}\right)^{2}\right)}\right) .
\end{aligned}
$$

Здесь $f\left(E_{n,+1}\right)=\left(\exp \left(\left(E_{n,+1}-\mu\right) / T\right)+1\right)^{-1}-$ функция распределения Ферми.

\section{3. Коэффициент поглощения электромагнитного излучения при учете электрон-примесного взаимодействия}

В [23] исследовано резонансное поглощение электромагнитного излучения электронами квантового канала с учетом рассеяния квазиодномерного электронного газа на ионизованной примеси.

Экранированный кулоновский потенциал в двумерном случае при $z=0$, используя разложение Фурье, имеет вид [24]

$$
\varphi(r, 0)=\int_{0}^{\infty} q A_{q}(0) J_{0}(q r) d q
$$

где $J_{0}(q r)$ - функция Бесселя нулевого порядка, $A_{q}(0)$ - коэффициент разложения, зависящий от $q$, $r=\sqrt{x^{2}+y^{2}}$ 
Матричный элемент $H_{R}$ выглядит точно так же, как и в предыдущем разделе. В области $r k_{0} \gg 0, \varphi(r)$ можно оценить [24]

$$
\varphi(r)=\frac{z e}{\varepsilon} \frac{1}{k_{0}^{2} r^{3}}
$$

где $k_{0}=1 / r_{0}, r_{0}-$ радиус экранирования, $z e-$ заряд примеси, $\varepsilon$ - диэлектрическая проницаемость.

Вычисление матричного элемента электрон-примесного взаимодействия приводит к выражению

$$
\begin{aligned}
& \left\langle n, k_{x}|\varphi(r)| n^{\prime}, k_{x}^{\prime}\right\rangle=\left(\frac{1}{\pi l_{c}^{2}}\right)^{1 / 2} \frac{1}{\sqrt{2^{n+n^{\prime}} n ! n^{\prime} !}} \frac{1}{L_{x}} \frac{z e}{\varepsilon} \\
& \times \int_{-\infty}^{\infty} \int_{-\infty}^{\infty} \exp \left(-i k_{x} x\right) \exp \left(-\left(y-y_{0}\right)^{2} / 2 l_{c}^{2}\right) \\
& \times H_{n}\left(\frac{y-y_{0}}{l_{c}}\right) \frac{1}{k_{0}^{2}\left(x^{2}+y^{2}\right)^{3 / 2}} \exp \left(i k_{x}^{\prime \prime} x\right) \\
& \times \exp \left(-\left(y-y_{0}^{\prime \prime}\right)^{2} / 2 l_{c}^{2}\right) H_{n^{\prime \prime}}\left(\frac{y-y_{0}^{\prime \prime}}{l_{c}}\right) d x d y,
\end{aligned}
$$

где $H_{n}(b)-$ полином Эрмита. Для интеграла по $x$ получим

$$
\int_{-\infty}^{\infty} \frac{\exp \left(-i k_{x} x\right) \exp \left(i k_{x}^{\prime \prime} x\right)}{k_{0}^{2}\left(x^{2}+y^{2}\right)^{3 / 2}} d x=\frac{2\left|k_{x}^{\prime \prime}-k_{x}\right|}{k_{0}^{2} y} K_{1}\left(y\left|k_{x}^{\prime \prime}-k_{x}\right|\right)
$$

где $K_{1}(b)-$ функция Макдональда.

Используем матричный элемент перехода между состояниями $|\alpha\rangle$ и $|\beta\rangle$ во 2-м порядке теории возмущений по электрон-фотонному и электрон-примесному взаимодействиям [23]:

$$
\langle\alpha|F| \beta\rangle=\sum_{v} \frac{\left\langle\alpha\left|H_{R}\right| v\right\rangle\langle v|V| \beta\rangle}{E_{\alpha}-E_{v}}+\sum_{v} \frac{\langle\alpha|V| v\rangle\left\langle v\left|H_{R}\right| \beta\right\rangle}{E_{\alpha}-E_{v}} .
$$

В нашем случае он имеет вид

$$
\begin{aligned}
& \left\langle n, k_{x}, 0,0|F|, n^{\prime}, k_{x}^{\prime},-\mathbf{f}, \mathbf{q}\right\rangle \\
& =\sum_{n^{\prime \prime} k_{x}^{\prime \prime}} \frac{\left\langle n, k_{x}, 0\left|H_{R}\right| n^{\prime \prime}, k_{x}^{\prime \prime}-\mathbf{f}\right\rangle\left\langle n^{\prime \prime}, k_{x}^{\prime \prime}|V| n^{\prime}, k_{x}^{\prime}\right\rangle}{E_{n,+1}-E_{n^{\prime \prime},+1}+\hbar \omega} \\
& \quad+\sum_{n^{\prime \prime} k_{x}^{\prime \prime}} \frac{\left\langle n, k_{x},|V| n^{\prime \prime}, k_{x}^{\prime \prime}\right\rangle\left\langle n^{\prime \prime}, k_{x}^{\prime \prime}, 0\left|H_{R}\right| n^{\prime}, k_{x}^{\prime}-\mathbf{f}\right\rangle}{E_{n^{\prime},+1}-E_{n^{\prime \prime},+1}-\hbar \omega} .
\end{aligned}
$$

Нахождение парциального КП проведем с использованием подхода, применяемого в [23]. Тогда можно получить

$$
\begin{aligned}
& K\left(n, n^{\prime}\right)=\frac{2 \pi \sqrt{\varepsilon}}{c \hbar N_{f}} N_{i}\left(\frac{1}{\pi l_{c}^{2}}\right) \\
& \times\left(\frac{1}{\sqrt{2^{n+n^{\prime}} n ! n^{\prime} !}} \frac{1}{L_{x}} \frac{z e}{\varepsilon}\right)^{2}\left|\frac{-i e \hbar}{m_{c y} l_{c}} \sqrt{\frac{2 \pi \hbar N_{f}}{\epsilon \omega}}\right|^{2} f_{0}\left(E_{n+}\right) \\
& \times \mid \int_{-\infty}^{+\infty} \frac{2\left|k_{x}^{\prime}-k_{x}\right|}{k_{0}^{2} y} K_{1}\left(y\left|k_{x}^{\prime}-k_{x}\right|\right) \exp \left(\left(y-y_{0}\right)^{2} / 2 l_{c}^{2}\right) \\
& \times \exp \left(\left(y-y_{0}^{\prime}\right)^{2} / 2 l_{c}^{2}\right) \mid \frac{\sqrt{\frac{n+1}{2}} H_{n+1}\left(\frac{y-y_{0}}{l_{c}}\right) H_{n^{\prime}}\left(\frac{y-y_{0}^{\prime}}{l_{c}}\right)}{\hbar \omega-\hbar \omega_{+}} \\
& -\frac{\sqrt{\frac{n^{\prime}}{2}} H_{n}\left(\frac{y-y_{0}}{l_{c}}\right) H_{n^{\prime}-1}\left(\frac{y-y_{0}^{\prime}}{\hbar l_{c}}\right)}{\hbar \omega_{+}} \\
& +\frac{\sqrt{\frac{n^{\prime}+1}{2}} H_{n}\left(\frac{y-y_{0}}{l_{c}}\right) H_{n^{\prime}+1}\left(\frac{y-y_{0}^{\prime}}{l_{c}}\right)}{\hbar \omega+\hbar \omega_{+}} \\
& -\left.\left.\frac{\sqrt{\frac{n}{2}} H_{n-1}\left(\frac{y-y_{0}}{l_{c}}\right) H_{n^{\prime}}\left(\frac{y-y_{0}^{\prime}}{l_{c}}\right)}{\hbar \omega+\hbar \omega_{+}}\right|^{2} d y\right|^{2} \\
& \times \delta\left(E_{n,+1}-E_{n^{\prime},+1}+\hbar \omega\right),
\end{aligned}
$$

где $N_{i}$ - число примесей, $f_{0}\left(E_{n,+1}\right)-$ функция распределения невырожденного электронного газа.

Рассмотрим далее переходы только из основного состояния $n=0$. Интеграл по $y$ имеет вид

$$
\begin{aligned}
\Lambda & =\int_{-\infty}^{\infty} \frac{2\left|k_{x}^{\prime}-k_{x}\right|}{y} K_{1}\left(y\left|k_{x}^{\prime}-k_{x}\right|\right) \\
& \times \exp \left(\left(y-y_{0}\right)^{2} / 2 l_{c}^{2}\right) \exp \left(\left(y-y_{0}^{\prime}\right)^{2} / 2 l_{c}^{2}\right) \\
& \times\left[\frac{\sqrt{\frac{1}{2}} H_{1}\left(\frac{y-y_{0}}{l_{c}}\right) H_{n^{\prime}}\left(\frac{y-y_{0}^{\prime}}{l_{c}}\right)-\sqrt{\frac{n^{\prime}}{2}} H_{n^{\prime}-1}\left(\frac{y-y_{0}^{\prime}}{l_{c}}\right)}{\hbar \omega-\hbar \omega_{+}}\right. \\
& \left.+\frac{\sqrt{\frac{n^{\prime}+1}{2}} H_{n^{\prime}+1}\left(\frac{y-y_{0}^{\prime}}{\hbar \omega+\hbar \omega_{+}}\right)}{l_{c}}\right] d y .
\end{aligned}
$$

Окончательно получим

$\frac{K\left(0, n^{\prime}\right)}{K^{0}}=\frac{1}{2^{n^{\prime}} n^{\prime} !} \sinh \left(\frac{\hbar \omega_{c}^{\prime}}{2 T}\right) \frac{\omega_{c}^{\prime}}{\omega} \frac{\exp \left(-\hbar \omega_{c}^{\prime} / 2 T\right)}{1+\tau_{0}^{2}\left(\left(\omega-\omega_{c}^{\prime} n^{\prime}\right)^{2}\right)}|\Lambda|^{2}$,

где

$$
K^{0}=\frac{8 \pi e^{6} z^{2} n_{e} \hbar N_{i} \tau_{0}^{2}}{c \varepsilon^{5 / 2} k_{0}^{4} L_{x}^{2}}
$$




\section{4. Заключение}

В работе найден КП электромагнитного излучения свободными носителями заряда в монослое форфорена. Рассмотрен как случай невырожденного, так и вырожденного электронного газа. На рис. 1 показана зависимость КП от частоты электромагнитного излучения. Отчетливо видны резонансные пики на кривой поглощения, которые возникают при условии равенства частоты излучения циклотронной частоте. Резонансы имеют лоренцовскую форму, обусловленную размытием уровней. Показаны (рис. 1) три резонансные кривые при различных значениях циклотронных частот.

Ясно, что зависимость КП от магнитного поля (циклотронной частоты) (рис. 2) также имеет резонанс-

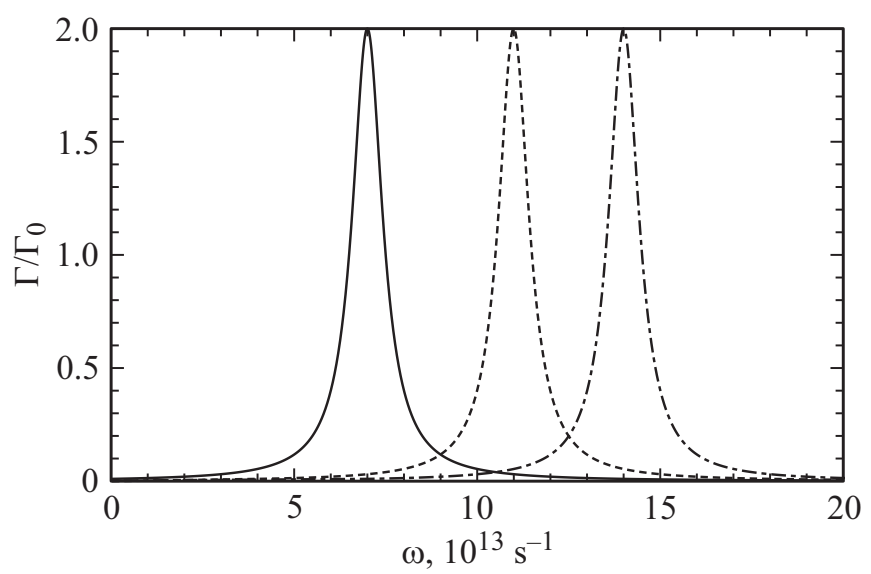

Рис. 1. Зависимость КП от частоты электромагнитного излучения в случае невырожденного электронного газа. $T=100 \mathrm{~K}, \omega_{c}=7 \cdot 10^{13} \mathrm{c}^{-1}, \tau=0.5 \cdot 10^{-13} \mathrm{c}$ - левая кривая; $T=100 \mathrm{~K}, \omega_{c}=11 \cdot 10^{13} \mathrm{c}^{-1}, \tau=0.5 \cdot 10^{-13} \mathrm{c}-$ средняя кривая; $T=100 \mathrm{~K}, \omega_{c}=14 \cdot 10^{13} \mathrm{c}^{-1}, \tau=0.5 \cdot 10^{-13} \mathrm{c}-$ правая кривая.

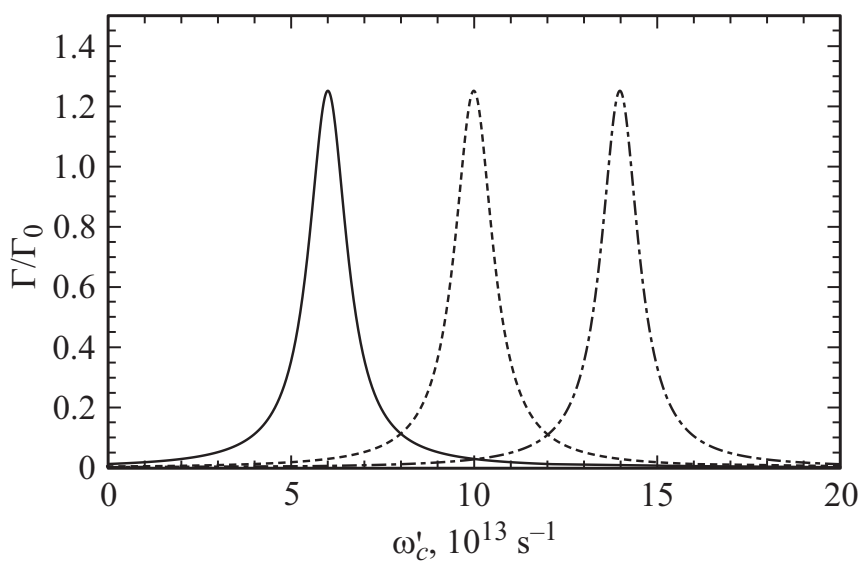

Рис. 2. Зависимость КП от циклотронной частоты в случае невырожденного электронного газа. $T=100 \mathrm{~K}$, $\omega=6 \cdot 10^{13} \mathrm{c}^{-1}, \tau=0.63 \cdot 10^{-3} \mathrm{c}-$ левая кривая; $T=100 \mathrm{~K}$, $\omega=10 \cdot 10^{13} \mathrm{c}^{-1}, \quad \tau=0.63 \cdot 10^{-13} \mathrm{c}-$ средняя кривая; $T=100 \mathrm{~K}, \omega=14 \cdot 10^{13} \mathrm{c}^{-1}, \tau=0.63 \cdot 10^{-13} \mathrm{c}-$ правая кривая.

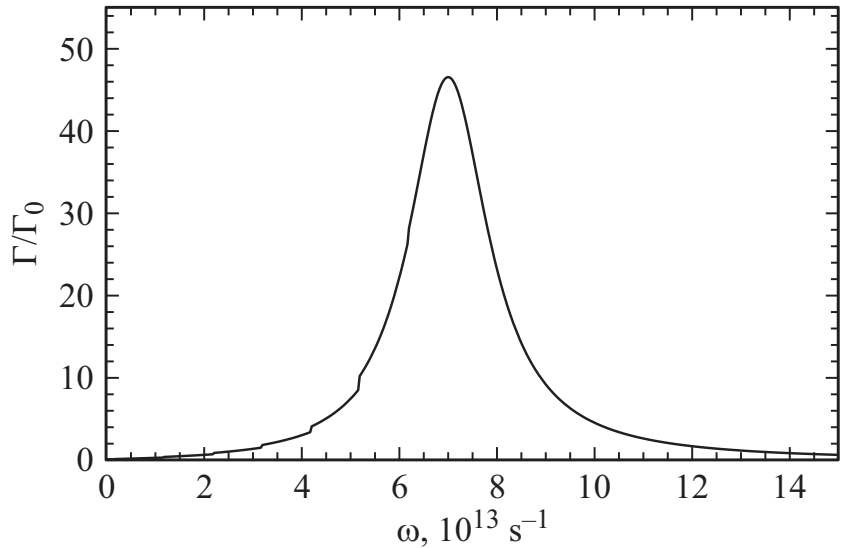

Рис. 3. Зависимость КП частоты электромагнитного излучения в случае вырожденного электронного газа. $T=1 \mathrm{~K}$, $\omega_{c}=7 \cdot 10^{13} \mathrm{c}^{-1}, \tau=0.5 \cdot 10^{-13} \mathrm{c}$.

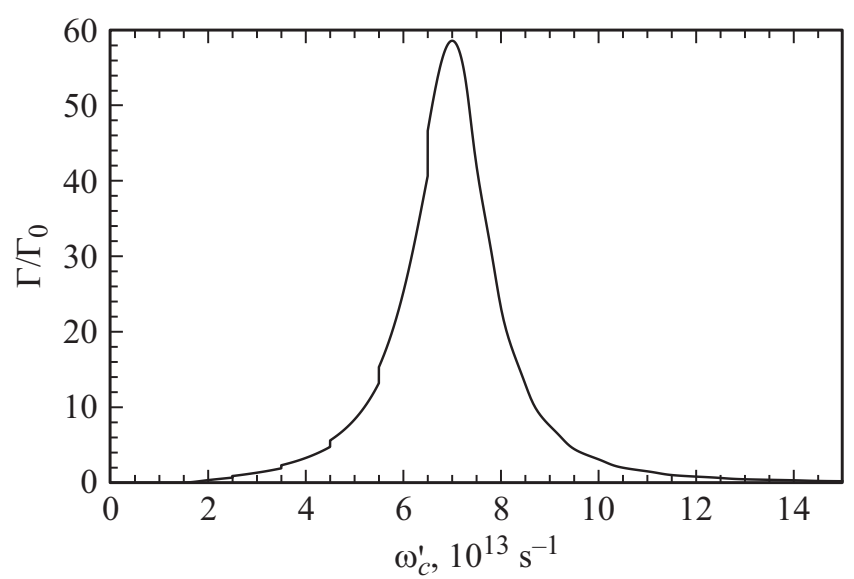

Рис. 4. Зависимость КП от циклотронной частоты в случае вырожденного электронного газа. $T=1 \mathrm{~K}, \omega=7 \cdot 10^{13} \mathrm{c}^{-1}$, $\tau=0.5 \cdot 10^{-13} \mathrm{c}$.

ное поведение. В формуле КП имеются множители, зависящие от магнитного поля, но они не так сильно влияют на форму резонансной кривой, как размытая рассеянием $\delta$-функция. Аналогично показаны три резонансные кривые (рис. 2) при различных значениях частот падающего электромагнитного излучения.

В случае вырожденного электронного газа зависимость КП от частоты электромагнитного излучения (рис. 3) имеет качественно другой вид. На кривой поглощения видны изломы. Это обусловлено тем, что когда энергия фотона достигает такого значения, что $\mu-\hbar \omega$ пересекается с энергетическим уровнем спектра $E_{n}$, происходит резкий скачок в плотности состояний. Наличие изломов на кривой $\Gamma(\omega)$ из-за скачков в плотности состояний ранее было отмечено для $3 D$ электронного газа в [22] и для $2 D$ электронного газа в квантовом цилиндре в [21]. Аналогичная ситуация наблюдается и в зависимости КП от циклотронной частоты (рис. 4). На кривой поглощения $\Gamma(\omega)$ видны изломы, обусловленные функциями Ферми. 


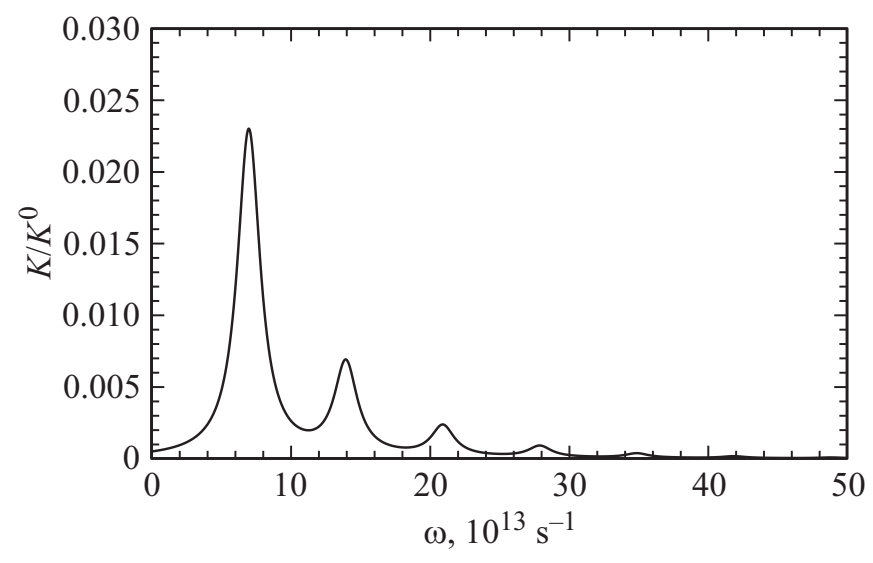

Рис. 5. Зависимость КП от частоты электромагнитного излучения при учете электрон-примесного взаимодействия в случае невырожденного электронного газа. $T=100 \mathrm{~K}$, $\omega_{c}=7 \cdot 10^{13} \mathrm{c}^{-1}, \tau=0.5 \cdot 10^{-13} \mathrm{c}$.

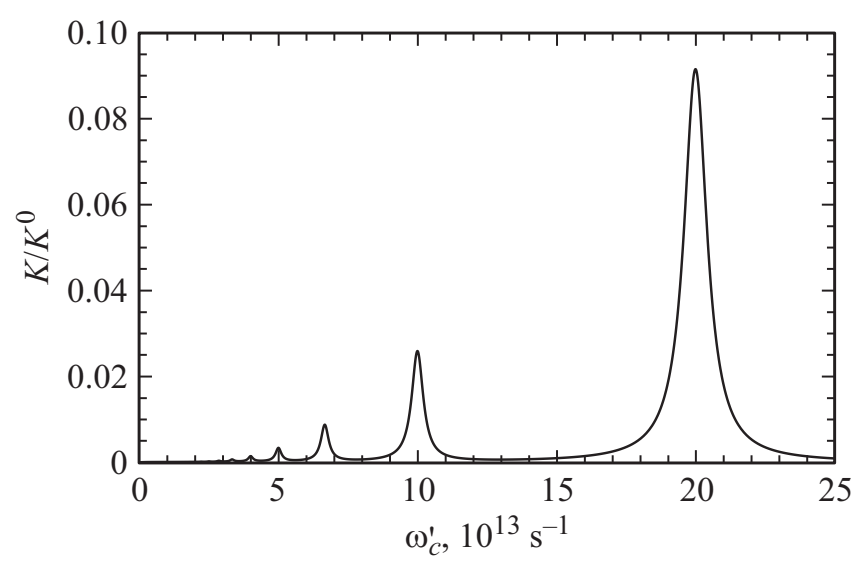

Рис. 6. Зависимость КП от циклотронной частоты при учете электрон-примесного взаимодействия в случае невырожденного электронного газа. $T=100 \mathrm{~K}, \omega=20 \cdot 10^{13} \mathrm{c}^{-1}$, $\tau=0.5 \cdot 10^{-13} \mathrm{c}$.

На рис. 5 показана зависимость КП от частоты электроманитного излучения при учете рассеяния электронов на ионизованной примеси. В случае невырожденного электронного газа видно множество резонансных пиков, имеющих лоренцову форму. Эти пики обусловлены забросами электронов на высокие энергетические уровни. Положение резонансных пиков определяется условием $E_{n+1}=E_{n}+\hbar \omega_{\text {res. }}$. На рис. 6 показана зависимость КП от циклотронной частоты при учете рассеяния электронов на ионизованной примеси. На графике четко видно, что с увеличением квантового числа $\mathrm{n}$ расстояние между пиками и интенсивность уменьшаются.

Диэлектрическая проницаемость $\varepsilon$ для многослойного фосфорена толщиной $\sim 10$ нм, помещенного в магнитное поле, исследовалась в [20]. Из результатов этой работы видно, что $\varepsilon(q)$ мало изменяется при характерных для поглощения излучения волновых векторах и практически не зависит от числа слоев фосфорена.
В связи с этим $\varepsilon$ так же, как и для полупроводникового $2 D$ электронного газа, можно считать постоянной величиной.

Если частота $\omega$ точно попадает в один из резонансов, то $\omega_{\text {res }}=\left(E_{n,+1}-E_{n^{\prime},+1}\right) \hbar^{-1}$. В этой точке без учета уширения уровней рассеянием $K\left(0, n^{\prime}\right)$ обращается в бесконечность.

Работа поддержана Российским фондом фундаментальных исследований (проект 16-02-00475).

\section{Список литературы}

[1] X.Y. Zhou, R. Zhang, J.P. Sun, Y.L. Zou, D. Zhang, W.K. Lou, F. Cheng, G.H. Zhou, F. Zhai, K. Chang. Sci.Rep., 5, 12295 (2015).

[2] M. Tahir, P. Vasilopoulos, F.M. Peeters. Phys. Rev. B, 92, 045420 (2015).

[3] J.M. Pereira, M.I. Katsnelson. Phys. Rev. B, 92, 075437 (2015).

[4] E. Taghizadeh Sisakht, F. Fazileh, M.H. Zare, M. Zarenia, F.M. Peeters. Phys. Rev. B, 94, 085417 (2016).

[5] Г.Т. Адамашвили. Письма ЖТФ, 44, 77 (2018).

[6] Ruimin Bai, Zheng Chen, Manman Gou, Yixin Zhang. Sol. St. Commun., 270, 76 (2018).

[7] Zongyu Huang, YanbingWu, Xiang Qi, Chaoyu He, Xiaohui Ren, Jianxin Zhong. Phys. Status Solidi B, 255, 1700370 (2018).

[8] Zhaohui Luan, Lei Zhao, Hao Chang, Dan Sun, Changlong Tan, Yuewu Huang. Superlat. Microstruct., 111, 816 (2017).

[9] Sandhya Chintalapati, Lei Shen, Qihua Xiong, Yuan Ping Feng. Appl. Phys. Lett., 107, 072401 (2015).

[10] A.R. Davletshin, S.V. Ustiuzhanina, A.A. Kistanov, D. Saadatmand, S.V. Dmitriev, Kun Zhou, E. Korznikova. Physica B: Condens. Matter, 534, 63 (2018).

[11] Tao Hu, Jisang Hong. J. Appl. Phys., 118, 054301 (2015).

[12] Marcin Kurpas, Martin Gmitra, Jaroslav Fabian. Phys. Rev. B, 94, 155423 (2016).

[13] Yuan Li, Xin Li, Qi Wan, R. Bai, Z.C. Wen. Physica E, 98, 33 (2018).

[14] Chuong V. Nguyen, Nguyen Ngoc Hieu, C.A. Duque, Doan Quoc Khoa, Nguyen Van Hieu, Luong Van Tung, Huynh Vinh Phuc. J. Appl. Phys., 121, 045107 (2017).

[15] Z.S. Popovic, Jamshid Moradi Kurdestany, S. Satpathy. Phys. Rev. B, 92, 035135 (2015).

[16] V.A. Saroka, I. Lukyanchuk, M.E. Portnoi, H. Abdelsalam. Phys. Rev. B, 96, 085436 (2017).

[17] J. Smotlacha, R. Pincak. Phys. Lett. A, 382, 846 (2018).

[18] Vy Tran, Li Yang. Phys. Rev. B, 89, 245407 (2014).

[19] Zhili Zhu, Chong Li, Weiyang Yu, Dahu Chang, Qiang Sun, Yu Jiaa. Appl. Phys. Lett., 105, 113105 (2014).

[20] Yongjin Jiang, Rafael Roldan, Francisco Guinea, Tony Low. Phys. Rev. B, 92, 085408 (2015).

[21] Н.Г. Галкин, В.А. Маргулис, А.В. Шорохов. ФТТ, 43, 511 (2001).

[22] Р.К. Баканас. ФТТ, 12, 3408 (1970).

[23] В.В. Карпунин, В.А. Маргулис. Опт. и спектр., 122, 1011 (2017).

[24] Tsuneya Ando, Alan B. Fowler, Frank Stern. Rev. Mod. Phys., 54, 437 (1982).

Редактор Г.А. Оганесян 


\title{
Resonant absorption of the \\ electromagnetic radiation in a monolayer phosphorene
}

\author{
V.V. Karpunin ${ }^{1}$, V.A. Margulis ${ }^{2}$
}

${ }^{1}$ Mordovian State Pedagogical Institute named after M.E. Evsevieva,

430007 Saransk, Russia

${ }^{2}$ Ogarev Mordovian State University,

430005 Saransk, Russia

\begin{abstract}
The absorption coefficient of the electromagnetic radiation in a monolayer phosphorene placed in a magnetic field is calculated. A degenerate and nondegenerate electron gas are considered. The resonance dependences of the absorption coefficient on the radiation frequency and on the applied magnetic field are demonstrated. If we taking account of electrons by ionized impurities scattering that leads to oscillatory dependences of the absorption coefficient on the radiation frequency and the external magnetic field. The resonance character of the absorption curve is shown. Conditions resonances and positions of resonant peaks are found.
\end{abstract}

